CP, 2018, Vol.7 - No13, pp. 9/26 ISSN 2014-6752. Girona (Catalunya). Universitat de Girona. TAMAYO ACEVEDO, Mónica Isabel y TAMAYO ACEVEDO, Lucía Stella: Los videojuegos para la comunicación en salud sexual de los escolares. Valoración de los profesores de secundaria. Recibido: 11/12/2017 - Aceptado: 12/3/2018

\title{
Los videojuegos para la comunicación en salud sexual de los escolares: Valoración de los profesores de secundaria
}

\section{Autoras \\ Mónica Isabel Tamayo Acevedo}

Profesora Titular, Investigadora. http://orcid.org/0000-0003-0404-6594.

Facultad de Comunicación. Universidad de Medellín. Colombia. Carrera 87 \#30-65, código postal 050026, Medellín, Antioquia, Colombia, (57)(4) 3405510

E-mail: motamayo@udem.edu.co.

\section{Lucía Stella Tamayo Acevedo}

Profesora Titular, Investigadora. http://orcid.org/0000-0003-4217-4423.

Escuela de Microbiología. Universidad de Antioquia. Colombia. Calle 67 \#53-108, código postal 050010, Medellín, Antioquia, Colombia, (57)(4) 2198332

E-mail: lucia.tamayo@udea.edu.co

\section{Resumen}

El aula como espacio vital para la educación sexual de los adolescentes, implica maestros capacitados en conocimientos y en estrategias de comunicación innovadoras que minimicen riesgos biológicos, psicosociales y culturales inherentes a la sexualidad e impacten en la deserción escolar y el bajo rendimiento académico. Objetivo: Caracterizar los conocimientos, importancia y uso en el aula que dan los profesores al videojuego comercial en procesos comunicativos para la educación sexual de adolescentes de Medellín. Metodología: Estudio descriptivo trasversal, participaron 52 profesores de secundaria respondiendo una encuesta sobre intervención en proyectos educativos en salud sexual, procesos comunicativos en el aula, conocimientos sobre uso de TIC por los estudiantes. Resultados. El 78,4\% intervenía en proyectos de educación sexual; el 90,6\% consideraron que los estudiantes recurren a internet para informarse; el $82,7 \%$ piensa que los educandos hacen uso irresponsable de

\section{Abstract}

The classroom as a vital space for adolescent sexual education, involves teachers trained in knowledge and innovative communication strategies that minimize biological, psychosocial and cultural risks inherent to sexuality and impact on school dropout and poor academic performance. Objective: To characterize the knowledge, importance and use in the classroom that teachers give to the commercial videogame in communicative processes for the adolescent sexual education of Medellín. Methodology: Cross-sectional descriptive study, involving 52 secondary school teachers responding to a survey on intervention in educational projects on sexual health, communicative processes in the classroom, knowledge about ICT use by students. Results: $78.4 \%$ intervened in sex education projects; $90.6 \%$ considered that students resort to the internet to inform themselves; $82.7 \%$ think that learners make irresponsible use of ICT; for $78.9 \%$ commercial video games communicate sexist, racist or violent ideas; $77.4 \%$ 
las TIC; para el 78,9\% los videojuegos comerciales comunican ideas sexistas, racistas 0 violentas; el $77,4 \%$ creen que los estudiantes juegan videojuegos para divertirse; el $66 \%$ estaría dispuesto a incluir los videojuegos como estrategia didáctica. Conclusiones: Los profesores de secundaria asumen alta participación en proyectos educativos en salud sexual, poseen una aproximación sobre el uso de los videojuegos por parte de sus educandos y estarían dispuestos a incorporar los videojuegos comerciales como estrategia didáctica para la comunicación asertiva en los procesos de prevención y promoción de la salud sexual.

Palabras clave: Comunicación en salud; Educación Sexual; Salud Sexual; Tecnologías de la Información y la Comunicación; Videojuegos believe that students play video games to have fun; $66 \%$ would be willing to include video games as a didactic strategy. Conclusions: Secondary teachers assume high participation in educational projects on sexual health, have an approach to the use of video games by their students and would be willing to incorporate commercial video games as a didactic strategy for assertive communication in prevention processes and promotion of sexual health.

Keywords: Communication in health; Sex Education; Sexual Health; Information and Communication Technologies; Videogames

\section{Introducción}

Con el ánimo de buscar nuevas estrategias pedagógicas, mediadas por la tecnología, para la enseñanza-aprendizaje de la comunicación y educación sexual de los adolescentes que les posibiliten la vivencia de una sexualidad sana, y a la vez intervengan las problemáticas de salud sexual, entre ellas, el embarazo no deseado, las infecciones de transmisión sexual -ITS- y las prácticas sexuales de riesgo, (Gallo y Molina, 2009),(Sandoval, Rodríguez, Garcia, 2008), la Facultad de Comunicación de la Universidad de Medellín y la Escuela de Microbiología de la Universidad de Antioquia, ambas de Colombia, ejecutaron el proyecto de investigación "Apropiación del videojuego comercial en procesos de comunicación en educación sexual, en profesores de secundaria de Medellín, 2015-2016", del cual se deriva el presente artículo que pretende Caracterizar conocimientos, importancia y uso en el aula que dan los profesores al videojuego comercial en los procesos comunicativos para la educación sexual de los adolescentes de Medellín.

En una búsqueda constante por articular escenarios educativos innovadores para potenciar el desarrollo de las habilidades que exige la sociedad del siglo XXI, y particularmente para equipar a los jóvenes para una sexualidad segura, respetuosa y con pleno consentimiento, diversas investigaciones resaltan la importancia del uso de herramientas digitales y entornos virtuales (Vásquez, Ferrer, 2015), de la apropiación y reflexión sobre espacios web que modelan ideas frente a lo que debe ser las expectativas y experiencias sexuales de los jóvenes (Reality \& Risk, 2018), y de la integración de nuevos productos culturales, como los videojuegos, en los procesos de enseñanza aprendizaje en el aula (Sánchez, 2013) (García, Cortés, Martínez, 2011) (Begoña Gros, 2009). 
En el caso particular de los videojuegos, autoras como Lacasa (2011), consideran que éstos pueden constituirse en instrumentos educativos para apoyar procesos de enseñanza-aprendizaje, siempre y cuando estén mediados por la reflexión. De igual forma, destacan las posibilidades que ofrecen los videojuegos para establecer relaciones entre distintos contextos de aprendizaje. Desde la perspectiva de López y Rodríguez (2016) los diferentes proyectos pedagógicos realizados en aulas españolas utilizando videojuegos comerciales dan como resultado experiencias muy positivas en cuanto a aumento de motivación de los estudiantes, desarrollo de habilidades, adquisición de conocimientos, potencialización de la imaginación, incremento de la atención y concentración; sin descartar las dificultades presentadas en el uso de éstos, como la falta de formación e inseguridad de los profesores para utilizarlos, y el inconveniente para articular el contenido de los videojuegos comerciales con los de las asignaturas.

Una de las falencias más reiterativas para la comunicación y educación en sexualidad para adolescentes, es la relacionada con la formación de los profesores (Martínez, Vicario, González, Baeza, 2014), en cuanto a conocimientos y estrategias de comunicación y educación para promover la salud sexual y prevenir situaciones problemáticas. Al no poseer los maestros, información y estrategias comunicativas y educativas pertinentes y más acordes a la realidad y cotidianidad tecnológica de los jóvenes, es poco el impacto de los programas de salud sexual (González, 2015).

La limitación en la formación de los profesores en el componente de salud sexual, acarrea la carencia de conocimientos científicos y estrategias innovadoras para informar y comunicarse de manera adecuada con los estudiantes en el acto educativo (Rosales, 2017), lo que incrementa las problemáticas comunicativas en el aula relacionadas con la educación sexual y la baja calidad de la educación en las instituciones educativas; llevando a la falta de acompañamiento y orientación basada en el conocimiento, y a la carencia de estrategias didácticas apoyadas en las TIC, caso los videojuegos comerciales, como recurso que permite no solo desarrollar competencias digitales sino también el aprendizaje significativo sobre los asuntos de la vida (Peralta, Zambrano, 2016)

Más allá de realizar intervenciones aisladas del sector salud en educación sexual con estrategias comunicativas y educativas tradicionales, el maestro es un actor fundamental para integrar en los proyectos de aula la educación sexual en la perspectiva de derechos sexuales y reproductivos y con enfoque de género (Rosales, 2017), identificar escolares vulnerables a la violencia sexual, brindar consejería y asesoría a estudiantes y sus familias y canalizar a los estudiantes a los servicios de salud.

A la par se deben reconocer las causas indirectas de este problema, tales como: la crisis en el sector educativo que afecta la calidad de la educación básica secundaria, el desprestigio por el cual pasa la labor docente que desmotiva a los maestros a realizar y comprometerse con programas de impacto social, la baja apropiación del sector salud y educativo de las TIC como estrategia metodológica en los procesos de comunicación, el acceso sin control de los adolescentes a los medios de comunicación, sobre todo la Internet que facilitan información no formativa en educación sexual, como es el caso de la pornografía (Jones, 2018), la falta de programas de salud sexual adscritos al escalafón docente, la carencia de capacitaciones flexibles en tiempos y en espacios virtuales para los maestros, el enfoque biologista que se tiene 
sobre la sexualidad y que se contrapone con la cultura de los adolescentes, el entorno familiar y social, el alto porcentaje de hogares con mujeres cabeza de familia o ambos padres trabajadores que no posibilita el acompañamiento en la formación de sus hijos, y el control cultural, social y político que se tiene sobre la sexualidad, entre otras. (Tamayo, Tamayo, Chávez, 2017) Pese a lo anterior, son escasas las investigaciones alusivas al conocimiento, percepción y el uso mismo de los videojuegos para implementarlos en el aula en la educación sexual de los escolares de secundaría. En este sentido, este trabajo parte de la hipótesis de que los profesores de secundaria tienen conocimiento sobre el uso de los videojuegos por parte de los estudiantes, establecen su importancia como estrategia didáctica en el proceso de enseñanza-aprendizaje en la comunicación y educación para la salud sexual y harían uso de éstos en el aula.

La importancia de este trabajo se soporta en otras investigaciones realizadas en Colombia sobre el comportamiento de las ITS, determinantes de la salud y factores de riesgo para la salud sexual de los escolares de Medellín (Villegas, Tamayo, 2016) y sobre el uso, apropiación y percepción de los adolescentes sobre los videojuegos comerciales con contenido sexual implícito (Tamayo, Tamayo, Chávez, 2017), las cuales en sus resultados tienen en común: el déficit en conocimientos en salud sexual de los estudiantes, la alta frecuencia de factores de riesgo para el inicio de relaciones sexuales a temprana edad, como son el consumo de alcohol, tabaco y sustancias psicoactivas, la percepción de su situación económica precaria, la insatisfacción y poca importancia que los adolescentes dan a su estudio, el gran porcentaje de estudiantes vinculados al trabajo informal, la alta frecuencia de factores de riesgo inherentes al comportamiento sexual, entre ellos el no uso del condón, relaciones sexuales con parejas diferentes a la formal, y tener parejas sexuales 10 años mayores a ellos.

En los tamizajes de ITS, realizados en escolares de Medellín (Villegas, Tamayo, 2016) se destaca, en el grupo de mujeres, la alta prevalencia para las infecciones por el virus del papiloma humano -PVH-, Chlamydia trachomatis, lesiones premalignas para cáncer de cuello uterino, candidiasis y vaginosis bacteriana. En hombres, la alta prevalencia de uretritis no gonococica y tricomoniasis; situación que es alarmante si se tiene en cuenta que la edad de los escolares fluctúo entre 11 y 19 años y que el promedio de edad de inicio de relaciones sexuales fue de 13 años para los hombres y 14 para las mujeres. Estas problemáticas en salud sexual afectan directamente la inasistencia y la deserción de los estudiantes por situaciones como el embarazo no deseado, el aborto, las crisis afectivas, el abuso sexual, la explotación sexual y las ITS. En consecuencia, es de vital importancia implementar en los programas de salud sexual dirigidos a adolescentes nuevas estrategias comunicativas y educativas para el aprendizaje significativo que integre el conocimiento como lo propone Blázquez Barba M, et al. (2018), con la actitud positiva al autocuidado y las practicas saludables.

\section{Método}

Se hizo un estudio descriptivo transversal, realizado durante el 2015 y 2016, con la participación de 52 profesores de secundaria de veintinueve instituciones educativas de Medellín-Colombia, asistentes a un diplomado bimodal -virtual y presencial- sobre "Educación Sexual para Docentes", en el que se abordaron temáticas relacionadas con la comunicación y educación en salud sexual.

Al inicio del diplomado se aplicó una encuesta auto-diligenciada, que incluía preguntas re- 
feridas a: - La caracterización sociodemográfica: edad, sexo, procedencia, lugar de vivienda, estrato socioeconómico, composición familiar; -Formación y experiencia docente: nivel de educación, cursos de capacitación recibidos, años de experiencia docente, cursos dictados en el área de salud sexual, proyectos desarrollados en salud sexual; - Educación sexual en el aula: estrategias utilizadas para brindar información y apropiación de conocimientos de salud sexual; y estrategias comunicativas y educativas y apropiación de TIC en el aula para el desarrollo de proyectos de salud sexual.

Los sesgos de selección se controlaron a través de los criterios de inclusión, los cuales fueron ser docente activo en secundaria y responder voluntariamente y de manera completa la encuesta; y se excluyeron profesores no activos, que ejercieran la docencia en la primaria básica, no respondieran de manera completa la encuesta o se negaran a hacerlo. Los sesgos de información se controlaron por medio de la prueba piloto realizada a cinco profesores que cumplieran los criterios de inclusión, más no estuvieran participando del diplomado, con el fin de revisar y evaluar el formulario en cuanto a la forma y estilo de las preguntas para la comprensión de las mismas en el contexto sociocultural de los y las participantes y con la estandarización de las investigadoras y auxiliares para orientar el auto-diligenciamiento de la encuesta, antes de dar inicio al desarrollo del diplomado. El tiempo aproximado empleado por los participantes para responder la encuesta fue de 40 minutos, sin incluir el tiempo de la lectura y aceptación del consentimiento informado por los participantes.

La información de las encuestas fue digitada por dos integrantes del equipo de investigación de manera independiente, para ello se elaboraron dos bases de datos en el programa Excel versión 7 que luego fueron cotejadas para detectar errores de digitación. El análisis estadístico de los datos se efectúo en el programa SPSS versión 21, para lo cual se utilizaron medidas de tendencia central y de dispersión, para las variables en nivel de medición de razón y frecuencias absolutas y relativas para las variables nominales y ordinales.

Está investigación no tienen ningún riesgo para la salud, por estar exenta de procedimientos invasivos, según la Resolución 008430 de 1993 del Ministerio de Salud de Colombia, (Minsalud, 2014). Se salvaguardó la privacidad de los profesores participantes, la diversidad cultural, religiosa, tendencia sexual e identidad de género. En las respuestas a las preguntas se incluyó la opción no deseo responder, si el profesor sintiera expuesta su vida privada. Se veló por los principios fundamentales de la ética en investigación: respeto, beneficencia, no-maleficencia y justicia.

\section{RESULTADOS}

\section{Descripción sociodemográfica y formación docente}

El rango de edad de los 57 participantes fluctúo entre 30 a 49 años, el 69,8\% eran mujeres, el $70,6 \%$ pertenecían a estratos socioeconómicos medio y medio-alto, el 37,7\% tenía formación de posgrado, el $66,6 \%$ poseían formación profesional y el 44,6\% licenciatura en educación. EI $42,6 \%$ contaba con experiencia docente entre 6 y 10 años.

Participación, capacitación, actividades realizadas y evaluación en los proyectos pedagógicos de educación para la sexualidad -PPES- 
El 78,4\% manifestó conocer el PPES institucional y el 44,2\% participaba en el desarrollo de este. Las funciones centrales de los profesores consistían en impartir formación, orientación y asistencia a los estudiantes. El 42,4\% manifestó no haber recibido inducción para ejecutar el PPES y el 69\% actualización en temas de salud sexual o inherente al PPES, en el último año.

Las actividades realizadas por los profesores en del desarrollo del PPES fueron: talleres, conversatorios y conferencias sobre autocuidado, 82,6\%; talleres, conversatorios y conferencias sobre salud sexual y reproductiva, $60,8 \%$; campañas de promoción y prevención, 56,5\%; la actividad menos ejecutada fue la asesoría en métodos anticonceptivos, el 78\% manifestó la necesidad de capacitación en esta temática. Se destaca que el 39\% piensa que es importante recibir capacitación en estrategias didácticas innovadoras para abordar diferentes temas relacionados con la salud sexual.

En relación con los recursos didácticos y tecnológicos que poseen las instituciones educativas para el desarrollo del PPES, el 53,1\% de los profesores manifestó contar con material para simulación, y el $67,6 \%$ con material audiovisual. El $29,4 \%$ se apoya en las TIC como recurso tecnológico. El $5,9 \%$ lo considera inadecuado, el $14,7 \%$ suficiente y el $35,3 \%$ no cuenta con este recurso.

El 39\% de los profesores expresó que la evaluación del PEES se hace anualmente, el 14,6\% dijo que no se evalúa y el 14,9\% no sabía si esta se hacía. La evaluación la realizaba el coordinador y equipo responsable del proyecto y los directivos de la institución, dejando con menor participación en este proceso a los estudiantes, los profesores y los padres de familia. Solo evaluaban actividades y cumplimiento de objetivos; no evalúan estrategias comunicativas y didácticas, deserción estudiantil, y rendimiento académico de los estudiantes en relación con el proyecto.

\section{Conocimientos de los profesores sobre uso de TIC por parte de los estu- diantes}

A la pregunta de la encuesta ¿A través de qué medios creen que los estudiantes reciben información sobre salud sexual? el $90,6 \%$ de los profesores consideran que lo hacen principalmente de internet; en este ítem también se incluyeron los libros, las cartillas, los periódicos las revistas y los videojuegos, a los cuales, ningún profesor hizo referencia. Igualmente, piensan que los estudiantes obtienen la información sobre temáticas de sexualidad, principalmente de sus congéneres, 90,6\%. Todos los profesores suponen que los estudiantes acuden inicialmente a sus amigos para hablar sobre sus situaciones personales de salud sexual y no con otras personas como padres y profesores. En este sentido, la confianza es depositada en los amigos.

El 82,7\% de los profesores consideró que los jóvenes hacen un uso no responsable de las TIC. Para el $78,9 \%$ de los profesores los videojuegos comerciales comunican ideas sexistas, racistas o violentas a los jugadores.

Sobre la regularidad con que los estudiantes utilizan diferentes tecnologías en la institución, el $40 \%$ de los profesores manifestaron que los estudiantes utilizan diariamente el computador 
y las tabletas con internet que les provee la misma institución; el 43,1\% manifestó que los estudiantes usan el computador y tableta personal con internet diariamente; el $78 \%$ considera que éstos hacen uso diario de su celular con internet y el $22,4 \%$ dicen que sus alumnos usan de máquinas de bolsillo. (Tabla 1)

Tabla 1. conocimientos de los profesores sobre el uso de tecnologías por los estudiantes

\begin{tabular}{|l|l|l|l|l|}
\hline CARACTERÍsTICA & No utilizan & Uso diario & Entre semana & No sabe \\
\hline $\begin{array}{l}\text { Computador y tabletas con } \\
\text { internet de la Institución } \\
\mathrm{n}=50\end{array}$ & $2 \%$ & $40 \%$ & $56 \%$ & $2 \%$ \\
\hline $\begin{array}{l}\text { Computador y tabletas con } \\
\text { internet personal } \\
\mathrm{n}=51\end{array}$ & $13.7 \%$ & $43.1 \%$ & $19.6 \%$ & $23.5 \%$ \\
\hline Celular con internet $\mathrm{n}=50$ & $6 \%$ & $78 \%$ & $8 \%$ & $8 \%$ \\
\hline Máquinas de bolsillo $\mathrm{n}=49$ & $18.4 \%$ & $22.4 \%$ & $12.2 \%$ & $46.9 \%$ \\
\hline
\end{tabular}

Fuente: Elaboración propia

Referente al uso que hacen los estudiantes de diferentes aparatos tecnológicos en actividades clase, el $82 \%$ de los profesores estima que los estudiantes hacen uso del computador con internet; el $33,3 \%$ que utilizan el computador y tableta con internet personal; para el $48 \%$ los estudiantes utilizan el celular en clase sin autorización del profesor. El 58\% de los profesores desconoce si los estudiantes utilizan máquinas de bolsillo. Para el $74.5 \%$ de los profesores el dispositivo que utilizan los estudiantes con mayor frecuencia es el Smarphone, el 68,6\% considera que es el Play Station, el 58,8\% estima que es el teléfono celular, y el 52,9\% el Xbox 360 . El $77,4 \%$ de los profesores cree que los estudiantes juegan videojuegos para divertirse, y el $26,6 \%$ para pasar el tiempo. Ninguno de los 53 profesores optó por las alternativas: mejorar el aprendizaje, adquirir habilidades motrices y relacionarse con otros jugadores. Sin embargo, en la pregunta sobre ¿cuáles habilidades cree pueden ser favorecidas en los jóvenes con el uso de videojuegos comerciales?, respondieron que desarrollan principalmente los reflejos, la agilidad mental y la atención y concentración de los estudiantes. Solo el 30,2\% de los profesores piensa que desarrolla la habilidad de comunicarse con los demás e incrementa la capacidad de análisis. (Gráfico 1) 


\section{GRÁFICO 1. Habilidades que consideran los profesores desarrollan los} videojuegos en los estudiantes

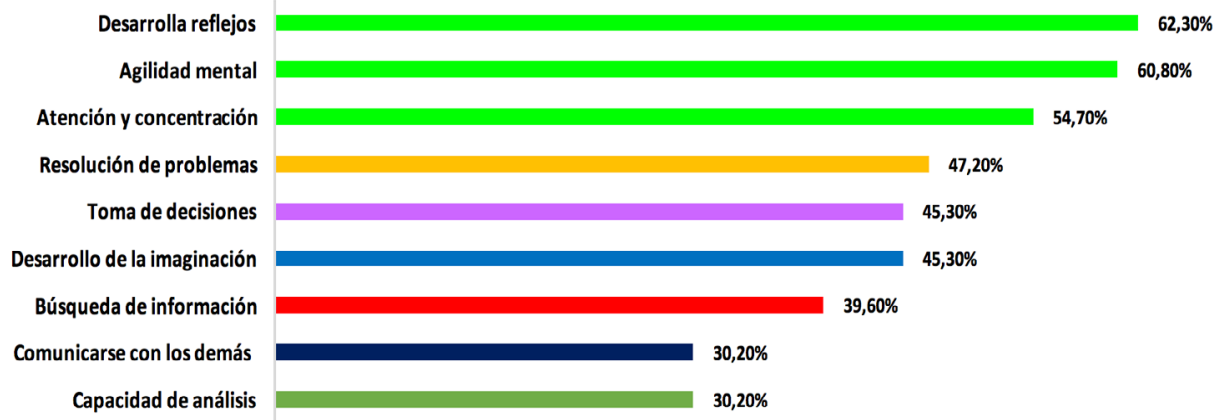

Fuente: Elaboración propia

Según los profesores, los videojuegos que más juegan los estudiantes son Mario Bross y Grand theft Auto San Andreas, seguido de Resident Evil. El 14,9\% no sabía qué jugaban los estudiantes. Para los profesores el mayor atractivo de los videojuegos para los estudiantes es superar el record de los amigos y el propio; el 7,5\% estima que lo menos atractivo es vivir romances. Para el $63,3 \%$ de los profesores, las relaciones que se dan entre los hombres y mujeres en los videojuegos son de rivalidad; para el $61,2 \%$ son de dominio; y solo el $22,4 \%$ piensa que son sexuales. (Gráfico 2)

\section{GRÁFICO 2. Relaciones que se dan entre los hombres y mujeres en los videojuegos que juegan los estudiantes}

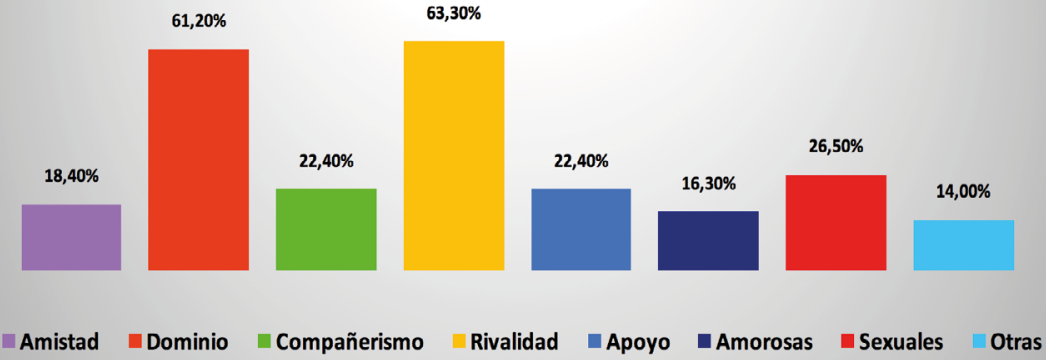

Fuente: Elaboración propia

El 75,5\% de los profesores consideró que la forma de vestir de los personajes en los videojuegos es una manifestación de contenido sexual; para el 63,5\% la apariencia de éstos es lo que se constituye en contenido sexual; y para el $60,4 \%$ el lenguaje corporal. Solo el $28,3 \%$ de los profesores piensa que los escenarios y escenografías bajo las cuales se mueven los personajes en el videojuego pueden ser una manifestación de contenido sexual. (Gráfico 3) 


\section{GRÁFICO 3. Manifestación de contenido sexual en los videojuegos que juegan los estudiantes}

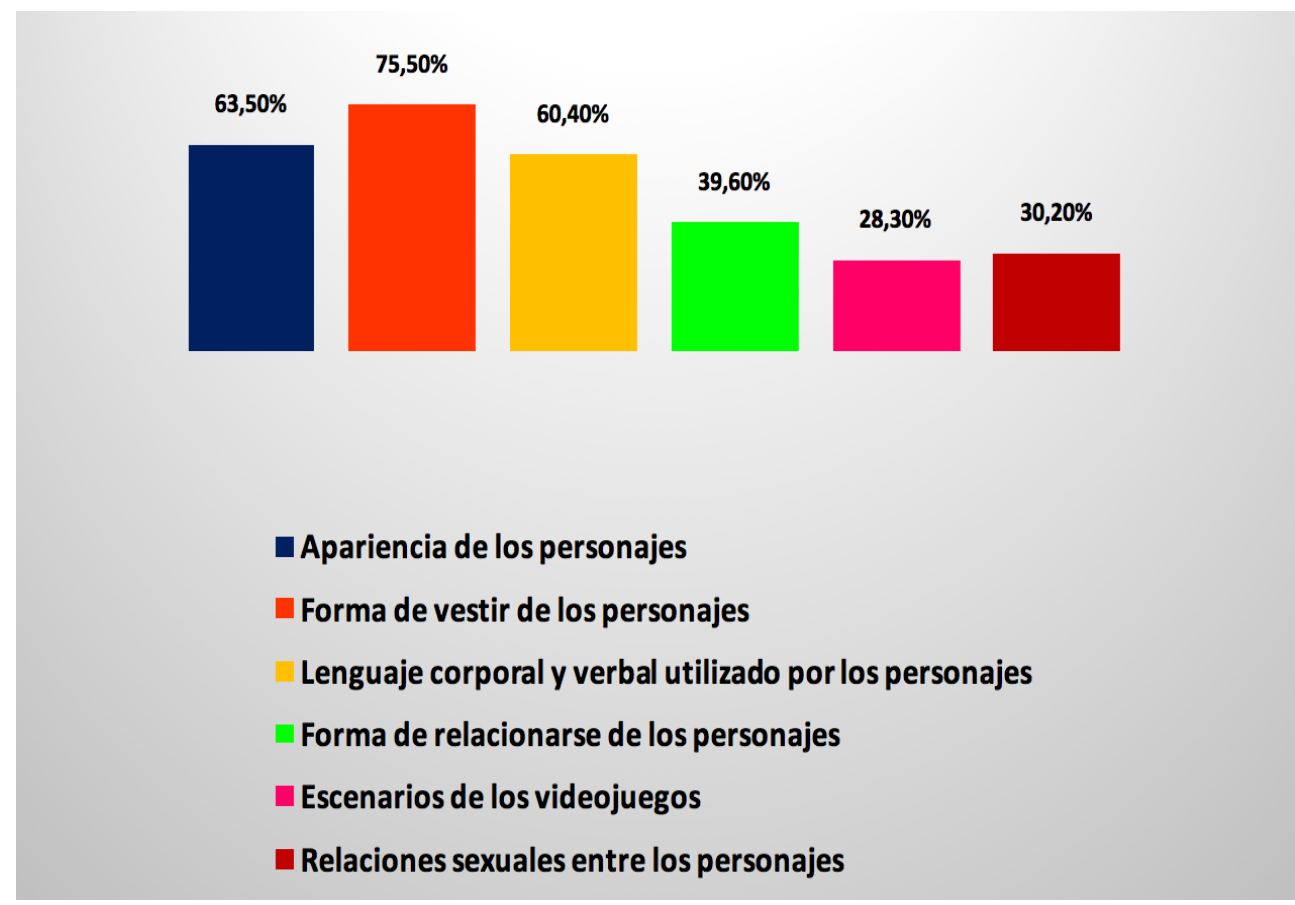

Fuente: Elaboración propia

\section{Percepción de los profesores sobre el uso de los videojuegos en el aula de clase \\ El 92\% de los profesores consideró el juego importante en los procesos de enseñanza-apren- dizaje, y el 78,4\% lo implementaría como estrategia pedagógica en el aula. El 71,2\% dijo que el videojuego comercial puede entrar en el ámbito educativo, y el $67,3 \%$ que éstos pueden ser una herramienta didáctica para mejorar la motivación de los estudiantes en los procesos de enseñanza-aprendizaje, el 66\% estaría dispuesto a incluirlos como estrategia didáctica del Proyecto Pedagógicos de Educación para la Sexualidad (PPES), teniendo como base funda- mental un buen acompañamiento y orientación del profesor, por lo que juzgan necesario reci- bir capacitación sobre su uso en el aula y el análisis de sus narrativas.}

Para los profesores los videojuegos son escenarios de reflexión, que posibilitan un análisis crítico y propositivo por parte de los estudiantes frente a diferentes temáticas, al igual que motivan y favorecen los ámbitos lúdicos para el aprendizaje. Sólo el $36,5 \%$ de los profesores juega videojuegos, el $73,5 \%$ conoce videojuegos de estrategia, el $69,4 \%$ de acción, el $63,2 \%$ de simulación, el $46,9 \%$ educativos, y el 10,2\% eróticos y sexuales. Para el 3,9\% el juego se opone a lo serio, yendo en contrasentido con los procesos educativos. Al hablar sobre las características pedagógicas del juego, los profesores opinan que éste tiene reglas propias, 63,3\%; que tiene límites de espacio y tiempo, 53,10\%; y que crea orden, $38,8 \%$. 
Ante la pregunta sobre la función del juego, el 73,5\% de los profesores consideró que éstos propician la competición, y el $46,9 \%$ que permiten descargar el exceso de energía. Para el $22,4 \%$, el juego permite prepararse para la realización de actividades cognitivas. El 14,3\% cree que el juego cumple con todas las funciones antes mencionadas, además de permitir adquirir dominio de si, propiciar la cooperación y relajarse.

\section{Discusión}

La alta participación y responsabilidad que tienen los profesores de este estudio en el desarrollo de los Proyectos Pedagógicos de Educación para la Sexualidad -PPES- en sus instituciones educativas, en los que deben impartir formación, orientación y asistencia a los estudiantes en temáticas relacionadas con la sexualidad, se contrapone a la insuficiente inducción para la ejecución de los mismos, al poco conocimiento y a la falta de actualización en temas sobre salud sexual.

Carencias que se ven reflejadas en el tipo de actividades que llevan a cabo para impartir la educación en salud sexual, y que vienen determinadas por estrategias educativas tradicionales que pueden interferir con el logro de los objetivos propuestos y el impacto de los programas, dadas las dinámicas de los jóvenes como nativos digitales. Situación ésta que se contrapone a las estrategias en comunicación en salud basadas en las nuevas tecnologías de comunicación, como el uso de internet para la toma de decisiones informadas e intercambio de experiencias en salud pública (Mosquera, 2002).

Es alarmante en este estudio, la situación relacionada con la evaluación del PEES, que en el mejor de los casos se evalúa anualmente, lo que limita la perspectiva en relación con el impacto que tienen, al igual que la toma de decisiones vinculadas con las temáticas a abordar, estrategias comunicativas y didácticas a utilizar en pro de un mejoramiento continuo. De igual forma es cuestionable la falta de participación en este proceso de los estudiantes, lo que se contrapone a la relevancia que dan autores como Blázquez Barba M, et al. (2018) sobre la importancia de participación de los jóvenes en la planificación, ejecución, seguimiento y evaluación de los programas de salud; refrendado con las teorías de comunicación participativa para las que el éxito de una campaña orientada al cambio de actitudes y comportamientos depende de la participación activa, en todo el proceso, de la comunidad afectada. (Mosquera, 2002)

Es significativo el porcentaje de profesores en este estudio que considera necesaria la capacitación en estrategias didácticas educativas innovadores para abordar temas relacionadas con la educación en salud sexual, y la apertura que evidencian a la incursión de dinámicas vinculadas con el uso de las TIC y con nuevos productos culturales, como el caso de los videojuegos, en los que ven grandes posibilidades para los procesos motivacionales del aprendizaje de los adolescentes, y en la preparación para la realización de actividades cognitivas.

Posiciones que se articulan con el pensamiento de Lacasa (2011) para quien la incursión del juego en la práctica educativa permite la ampliación de los escenarios de aprendizaje y enseñanza asociados a los videojuegos; y con la de Masas (2017), en cuya indagación sobre las características y oportunidades que ofrece el videojuego como dispositivo educativo encuentra que éstos proporcionan un entorno de aprendizaje rico y complejo, pero en el que se hace 
necesario modificar las estrategias educativas y contar con un profesor abierto a explorar este universo, para así lograr una integración coherente y adecuada en el aula de clase. Pese a lo anterior, en este estudio las estrategias educativas para acercar el conocimiento en salud sexual están enmarcadas en talleres, conversatorios, asesorías y conferencias.

Para el 90,6\% de los profesores de este estudio, los estudiantes reciben información en temáticas en salud sexual principalmente de internet, lo que se correlaciona con el estudio realizado por Blázquez Barba M., et al. (2018), sobre el uso de internet por los adolescentes en la búsqueda de información sanitaria, en la que el $53,6 \%$ de los jóvenes buscaba información en salud a través de Internet, y de éstos el 49,6\% lo hacían sobre enfermedades de trasmisión sexual y $\mathrm{VIH}$, y el 21,5\% sobre embarazo y anticoncepción. Y con el estudio realizado por Guillamón y Martínez (2012) sobre uso de Internet y redes sociales para la salud en jóvenes, en el que el $100 \%$ de los encuestados usaba Internet de forma frecuente y el $75 \%$ lo hacía para buscar información sobre salud, particularmente en temas relacionados con la sexualidad, las drogas y los problemas de alimentación.

Resultados que se orquestan con la idea planteada por Begoña Gros (2012) sobre la importancia de incorporar las TIC en la práctica educativa, haciendo uso cotidiano de éstas en las diversas áreas de formación, y en el caso concreto del uso de los videojuegos comerciales integrándolos desde la perspectiva de apoyo al aprendizaje.

Se debe tener en cuenta que los videojuegos educativos, a diferencia de los comerciales, son diseñados con el objetivo de transmitir contenidos curriculares, lo que limita los contextos de la experiencia y la inmersión, por lo tanto, el profesor debe convertirse en un experto en utilizar las características del videojuego comercial de forma educativa, seleccionando los que sean más pertinentes para el logro de los objetivos pedagógicos (Begoña Gros, 2012). En esta selección de videojuegos comerciales, es importante retomar los treinta y seis principios de aprendizaje incorporados en su diseño, que para Gee (2004) facilitan el proceso de enseñanza. Principios como el semiótico, que permite aprender y apreciar las interrelaciones dadas en el juego; el de moratoria social, que posibilita asumir riesgos en un espacio en el que están amortiguadas las consecuencias del mundo real; o el de transferencia, que permite que lo aprendido en el juego pueda ser aplicado después.

Otro resultado de la investigación muestra que el 82,7\% de los profesores piensa que los jóvenes no hacen un uso responsable de las TIC. Lo que se contrasta con el estudio realizado por Bringué y Sádaba (2010) en el que se pone de manifiesto que más del 70\% de los menores españoles entrevistados, declararon haber aprendido por si mismos a usar internet, lo que para los autores se constituye en un ejemplo claro de autonomía frente al uso de la tecnología y en un reto para la formación educativa de los docentes en esta área, de modo que los jóvenes puedan percibir algún tipo de autoridad en los adultos frente a esta. En sus conclusiones, Bringué y Sádaba (2010) apelan a los educadores para que creen pautas educativas integrales que permitan desarrollar el uso responsable, seguro y fructífero de las TIC.

La idea sobre el uso no responsable de las TIC que tienen los profesores de este estudio podría cambiarse si se tienen en cuentan los planteamientos de Massa (2017) sobre la importancia de la inclusión de las TIC en ambientes de aprendizaje desde los aspectos no solo tecnológicos sino también pedagógicos, sustentados en principios como la problematización de lo apren- 
dido, la integración del saber, el multiculturalismo, la prospectiva, la transferencia, entre otros; y en los Begoña Gros (2009) sobre la necesidad de incorporar las TIC, particularmente los videojuegos, en el aula bajo la guía y acompañamiento del profesorado, quien debe incidir en la transformación de la experiencia de juego en una experiencia reflexiva, y en la determinación de los aprendizajes del juego.

En el caso del uso de videojuegos comerciales en el aula para abordar temáticas relacionadas con la salud sexual es importante aclarar que muchos de ellos muestran y fomentan roles o estereotipos sexistas, racistas y violentos, por lo que el profesor debe abrir espacios de diálogo con sus estudiantes para acompañarles en el desarrollo de un sistema de valores que les permita enjuiciar este tipo de expresiones (Montero, Ruíz, Díaz, 2010).

La investigación realizada por Bringué y Sádaba (2010) muestra el equipamiento tecnológico que poseen los niños y jóvenes en España, el $97 \%$ posee PC, el $82 \%$ internet, el 57\% portátil, lo que evidencia un mayor acceso y uso a la tecnología por las nuevas generaciones, para estudiar, jugar, relacionarse y entretenerse. Datos que se correlacionan con los de este estudio, en el que los profesores expresan que el $43,1 \%$ de los estudiantes utilizan diariamente computador y tableta personal con internet. Para Bringué y Sádaba (2010) el acceso a la tecnología les permite a los niños y jóvenes vivir emancipados dentro de su hogar en relación a consumo de medios, tiempo y espacio de uso, y servicios y contenidos accedidos.

Otro hallazgo se relaciona con la percepción que tienen los profesores sobre para qué juegan los estudiantes, el 77,4\% piensa que para divertirse, entrando así en la misma dinámica de resultados dados por Alonqueo y Rehbein (2008) en su investigación sobre usuarios habituales de videojuegos, en la que el $75 \%$ de los jóvenes participantes señaló que jugaba sólo para divertirse.

Las habilidades que los profesores de este estudio consideran desarrollan los estudiantes al hacer uso de videojuegos comerciales fueron los reflejos, 62,3\%; la agilidad mental, 60,8\%; y la atención y concentración, $54,7 \%$. Resultados compatibles con la percepción que tienen los jóvenes videojugadores sobre las habilidades que se adquieren al jugar, en el estudio realizado por Alonqueo y Rehbein (2008), según el cual la agilidad mental es la que más se desarrolla, 86\%; la creatividad y la resolución de problemas, $71 \%$; y la habilidad para tomar decisiones, $70 \%$. Y con las conclusiones a las que llegan López y Rodríguez (2016) en su revisión bibliográfica sobre investigaciones relacionadas con el juego y el videojuego en educación, en las que se apunta que los videojuegos resultan de gran utilidad en los procesos de aprendizaje porque implican activamente a los estudiantes, ayudan a focalizar la atención, potencian la habilidad para resolver problemas, ayudan en la memorización y en el desarrollo del razonamiento deductivo.

Lo que en términos generales refuerza la idea de Gee (2004) sobre los videojuegos como potenciadores del razonamiento inductivo-deductivo y de variadas habilidades cognitivas para la comprensión y valoración del entorno social y cultural.

En este estudio el 30,2\% de los profesores piensa que los videojuegos desarrollan en el estudiante la habilidad de comunicarse e incrementar la capacidad de análisis, reconociendo así, como lo plantea Gee (2004) el potencial que tienen para conducir al aprendizaje activo y 
crítico; y sus posibilidades para el desarrollo de competencias sociales y ciudadanas, bajo experimentación de valores de trabajo colectivo, de conocimiento propio y de otras realidades (Montero, Ruíz, Díaz, 2010)

El 78,9\% de los profesores consideró que los videojuegos comerciales comunican ideas sexistas, racistas o violentas a los jugadores, resultado que se relaciona con los de la investigación realizada por Díez (2009) sobre videojuegos y sexismos, centrada en los contenidos y valores que promueven los videojuegos comerciales, en los que se evidencian que la representación física de los personajes femeninos en los videojuegos atiende a la exageración, con idealizaciones tomadas frecuentemente de personajes del cine erótico (Jones, 2018), y en el 73\% de los casos la vestimenta de éstos no responde a la historia del juego sino a la insinuación y seducción ante la mirada masculina.

De igual forma, Díez y Terrón (2014) expresan que los videojuegos contribuyen profundamente a una socialización hacia la violencia de género, situación que genera un fuerte impacto sobre la imagen que las niñas y adolescentes construyen de ellas mismas y sobre las pautas de comportamiento que asumen los jóvenes respecto a la mujer, basados en una visión estereotipada y limitada de lo femenino.

En este estudio el $71,2 \%$ de los profesores consideró que el videojuego comercial puede entrar en el ámbito educativo, y el 67,3\% el ser una herramienta didáctica para mejorar la motivación de los estudiantes, posición que se ajusta a los planteamientos de Montero, Ruíz y Díaz (2010), para quien los videojuegos son herramientas educativas que forman parte de la cultura de la imagen del siglo XXI, ofreciendo un aprendizaje por ensayo-error, no lineal y en contextos significativos; y a la de Gee (2004) sobre el videojuego como otra forma de aprender y pensar diferente a la acostumbrada dentro del sistema educativo. Idea reforzada por Etxeberria (2012) al plantear la necesidad de una reflexión sobre los videojuegos en el ámbito educativo, tanto desde sus aspectos preventivos como de su utilización en la escuela.

Frente a la concepción que tienen los profesores participantes en esta investigación sobre el valor educativo de los videojuegos, se destaca que el $36,5 \%$ son videojugadores, y el $96,1 \%$ cree que los videojuegos no se oponen a lo serio, resultados que se contraponen a los presentados por Sánchez (2013) en su estudio sobre la incorporación de los videojuegos en el aula como recurso didáctico, en el que el 94,3\% de los profesores consideró los videojuegos un distractor, y el $100 \%$ no había jugado videojuegos, ni con sus hijos. Desde la perspectiva de Etxeberria (2012) los videojuegos están invadiendo el mundo educativo, y los docentes siguen ajenos a estos y su aprovechamiento para mejorar y motivar el aprendizaje de los estudiantes. En el estudio realizado por Alonqueo y Rehbein (2008) los jóvenes participantes juzgan que los valores que se potencian con los videojuegos son la competición en un $89 \%$ para los hombres y un $92 \%$ para las mujeres, lo que se correlaciona con la percepción que tiene el $73,5 \%$ de los profesores, en esta investigación, sobre los videojuegos como propiciadores de la competición.

\section{Conclusiones}

Los profesores participantes en este estudio cuentan con un buen conocimiento del Proyecto Pedagógico de Educación para la Sexualidad -PPES-, que responde al cumplimiento de la normatividad colombiana sobre derechos humanos sexuales y reproductivos para la sexualidad. 
Pese a lo anterior, es preocupante la situación sobre el insuficiente tiempo e inducción para la ejecución del mismo, la falta de un proceso evaluativo continuo de este, la poca actualización que reciben en temas de salud sexual y la carencia de estrategias didácticas para abordarlos utilizando recursos tecnológicos propios del siglo XXI. La predominancia de la función formativa, de orientación y asistencia que hacen los profesores a los estudiantes justifica su capacitación en temas de educación sexual a fin de que puedan cumplir de forma efectiva con éstas.

La conciencia que tienen los profesores sobre el alto uso de tecnología en el aula, y fuera de ella, por parte de los estudiantes; el uso recreativo de las TIC -videojuegos, redes sociales, etc.como una actividad que no afecta negativamente el desarrollo psicosocial de los estudiantes; el uso poco responsable que éstos hacen de ellas; el internet como el medio más utilizado por los jóvenes para recibir información sobre salud sexual; la idea de que los videojuegos comerciales comunican ideas sexistas, racistas y violentas a los jugadores; se constituyen en factores importantes para determinar la capacitación a los profesores para que hagan uso de las TIC, particularmente de los videojuegos, en los procesos comunicativos y de enseñanza-aprendizaje de la salud sexual, bajo una mirada crítica y reflexiva en un diálogo continuo maestro-estudiante. Lo anterior se debe articular con la adquisición y adecuación de aulas tecnológicas en las instituciones.

Pese a que un alto porcentaje de profesores cree que los estudiantes utilizan los videojuegos solo con fines lúdicos, desconocen el potencial que tienen en el desarrollo de habilidades motoras, en procesos de socialización a partir de juegos colaborativos, y en el aprendizaje de diferentes áreas de conocimiento, están dispuestos a utilizarlos como recurso y estrategia pedagógica en el aula, particularmente para trabajar sus proyectos de educación para la sexualidad, por considerar sus narrativas escenarios propicios para la reflexión y análisis crítico, y por su carácter interactivo e inmersivo que motivan y favorecen los ámbitos lúdicos para el aprendizaje. Esta apertura a nuevas formas de enriquecer los procesos de enseñanza-aprendizaje, debe sustentarse en un buen proceso de capacitación sobre el uso de videojuegos comerciales en el aula y análisis de sus narrativas.

\section{Referencias bibliográficas}

- $\quad$ Alonqueo, B., Rehbein, F. (2008) Usuarios habituales de videojuegos: una aproximación inicial. Última Década, 16(2), 11-27. doi: http://dx-doi.org/10.4067/S0718-22362008000200002.

- Begoña Gros, S. (2009). Certezas e interrogantes acerca del uso de los videojuegos para el aprendizaje. Revista Comunicación, 1(7), 251-264. ISSN: 1989-600X. Recuperado de: http://www.revistacomunicacion.org/pdf/n7/articulos/a17 Certezas e interrogantes acerca del uso de\%20 los videojuegos para el aprendizaje.pdf

- Begoña Gros, S. (2012). Estrategias de aprendizaje basado en videojuegos en la formación del profesorado. Actas I Congreso Internacional de Videojuegos y Educación. 5-15. Recuperado de: https://www.uv.es/ordvided/ACTAS/ACTAS\%20CIVE\%202012.pdf

- Blázquez Barba M, et al. (2018) Uso de internet por adolescentes en la búsqueda de información sanitaria. Aten Primaria. 2018. doi: https://doi.org/10.1016/j.aprim.2017.06.005

- Bringué, X., Sádaba, C. (2010). Niños y adolescentes españoles ante las pantallas: rasgos configuradores de una generación interactiva. CEE Participación Educativa, 15, 86-104. Recuperado de: http://dadun.unav.edu/bitstream/10171/18443/1/n15-sadaba-chalezquer.pdf 
- Díez Gutiérrez, E. J. (2009). Videojuegos y sexismos: innovación tecnológica y consolidación de un modelo social desigualitario. Aequalitas: Revista jurídica de igualdad de oportunidades entre mujeres y hombres, 24, 56-68. Recuperado de: https://dialnet.unirioja.es/ ejemplar/234047

- Díez Gutiérrez, E.J., Terrón Bañuelos, E. (2014). Sexismo y violencia de género en videojuegos. Revista Científica ESEC. Suplemento Exedra de 2014 Sexualidade, género e educação. 221-232. Recuperado de: http://www.exedrajournal.com/wp-content/uploads/2014/12/ sup14-221-232.pdf

- Etxeberria, B. (2012). Videojuegos: riesgos y oportunidades en la educación. Actas I Congreso Internacional de Videojuegos y Educación. 22-31. Recuperado de: https://www. uv.es/ordvided/ACTAS/ACTAS\%20CIVE\%202012.pdf

- Gallo, N., Molina, A. (2009). Evaluación de impacto proyecto salud sexual y reproductiva de adolescentes en Medellín. "Sol y Luna" componente cualitativo, 2008. Revista Salud Pública de Medellín, 4(1),19-32.

- García, M., Cortés, S., Martínez, R. (2011). De los videojuegos al currículum escolar. Las estrategias del profesorado. Revista Icono 14, 2, 249-261. ISSN 1697-8293.

- Gee, J. (2004). Lo que nos enseñan los videojuegos sobre el aprendizaje y el alfabetismo. Málaga, España: Coleccion Aulae.

- González, Y (2015). El papel del docente en la educación para la sexualidad: algunas reflexiones en el proceso educativo escolar. Revista Actualidades Investigativas en Educación. Costa Rica. Vol. 15 Número 3 Septiembre-Diciembre. Pp. 1-15. doi: http://dx.doi. org/10.15517/aie.v15i3.20335.

- Guillamón, N.; Martínez, M. (2012). Uso de internet y las redes sociales para la salud en adolescentes: Evaluación de necesidades para un servicio online de salud mental. En: Pérez Gómez, L., et. al. (Coord.). Tecnologías de la comunicación, jóvenes y promoción de la salud. España: Gobierno de La Rioja, Consejería del Gobierno de La Rioja, Rioja Salud.

- Jones, M. (11 de febrero de 2018). Aprender de la Pornografía. New York Times. Recuperado de: https://www.nytimes.com/es/2018/02/11/lo-que-los-adolescentes-aprenden-dela-pornografia/

- Lacasa, P. (2011) Los videojuegos. Aprender en mundos reales y virtuales. Madrid: Ediciones Morata, S. L.

- López, S.; Rodríguez, J. (2016) Experiencias didácticas con videojuegos comerciales en las aulas españolas. Revista DIM: Didáctica, Innovación y Multimedia. No. 33, 2016. ISSN-e 1699-3748.

- Martínez, J.L., Vicario, I., González, P., Baeza, I. (2014). Infancia y Aprendizaje. Journal for the Study of Education and Development, 37(1), 131-148. ISSN 0210-3702, ISSN-e 1578-4126.

- Massa, S. (2017) Videojuego en el Aprendizaje. Oportunidades y Desafíos. Prometeica Revista de Filosofía y Ciencias- No. 15-2017. ISSN: 1852-9488.

- Montero, E., Ruíz, M., Díaz, B. (2010). Aprendiendo con videojuegos: Jugar es pensar dos veces. Madrid: Narcea, S.A. De Ediciones.

- Mosquera, M. (2002) Comunicación en Salud: Conceptos, Teorías y Experiencias. Disponible en: http://www.comminit.com/la/content/comunicaci\%C3\%B3n-en-salud-conceptos-teor\%C3\%ADas-y-experiencias

- Peralta, P.A., Zambrado, E.C. (2016) Educación para la sexualidad con estrategias didácticas TIC, en adolescentes de 14 a 16 años en instituciones educativas oficiales de básica secundaria. Revista Linhas. Florianópolis, v. 17, n. 33, p. 135-157, jan./abr. 2016. 
doi: 10.5965/1984723817332016135 http://dx.doi.org/10.5965/1984723817332016135

- Reality \& Risk. (2018). It's time we talked. Australia: http://www.itstimewetalked.com. au/

- Resolución número 8430 de 1993. Octubre 4 de 1993. Minsalud. Colombia. (2014). Disponible en: https://www.minsalud.gov.co/Normatividad Nuevo/RESOLUCION\%20 8430\%20DE\%201993.pdf

- $\quad$ Rosales, A.L. (2017). Educación sexual y género en primarias mexicanas ¿qué dicen los libros de texto y el profesorado?. Revista Electrónica Educare (Educare Electronic Journal) EISSN: 1409-4258 Vol. 21(2) Mayo-Agosto, 2017: 1-21 [Número publicado el 01 de mayo del 2017] Universidad Pedagógica Nacional México

- Sánchez, M. (2013). Profesores frente a los videojuegos como recurso didáctico. Revista Didáctica, Innovación y Multimedia (DIM), 9(25). Abril 2013. ISSN 16993748.

- Sandoval, J., Rodríguez, M., Garcia, G. (2008). Salud sexual y reproductiva en adolescentes de Medellín, 2006. Revista Salud Pública de Medellín, 2(1), 7-25.

- $\quad$ Tamayo, L., Tamayo, M., Chávez, Ma. (2017). Uso y percepción de videojuegos comerciales con contenido sexual implícito por adolescentes. Colombia, 2014. Revista Adolescência \& Saúde, 14(4).

- Vásquez Cano, E., Ferrer Delgado, D. (2015) La creación de videojuegos con Scratch en Educación Secundaria. Revista Communication Papers, № 6, páginas 63 a 73. Departamento de Filología y Comunicación de la Universidad de Girona. Recuperado el 27 de febrero de 2018 de: http://www.communicationpapers.es

- Villegas, A., Tamayo, L. (2016). Prevalencia de infecciones de transmisión sexual y factores de riesgo para la salud sexual de adolescentes escolarizados, Medellín, Colombia, 2013. Revista IATREIA, 29(1), 5-17. doi: http://dx.doi.org/10.17533/udea.iatreia.v29n1a01

\section{CURRICULUM VITAE \\ LUCIA STELLA TAMAYO ACEVEDO.}

Doctora en Ciencias Médicas, Universidad de Colima- México. Mg. en Epidemiología y licenciada en enfermería, Universidad de Antiquia-Colombia. Profesora Titular en la Escuela de Microbiología,Universidad de Antioquia. Coordinadora del grupo de Investigación Salud Sexual y Cáncer. Ha desarrollado proyectos de investigación con enfoques biomédicos, epidemiológicos y cualitativos, relacionados con la promoción de la salud y la educación en salud y la prevención de enfermedades de interés para la salud publica, en adolescente, mujeres en edad reproductiva y personas con VIH. Ha publicado diversos artículos y capítulos de libros sobre las temáticas de sus investigaciones. 